\title{
Intraoperative microvascular Doppler monitoring of blood flow within a spinal dural arteriovenous fistula: a precious surgical tool
}

\author{
Case report \\ Domenico Gerardo Iacopino, M.D., Maria Giusa, M.D., Alfredo Conti, M.D., \\ Salvatore Cardali, M.D., and Francesco Tomasello, M.D.
}

Neurosurgical Clinic, University of Messina, Messina, Italy

\begin{abstract}
The authors describe a case of spinal arteriovenous fistula (AVF) treated by a microvauscular Doppler-assisted surgical interruption of the arterialized vein. Microvascular Doppler monitoring represents a valid, widely available, noninvasive tool that enables identification, through flow spectrum analysis, of components of this type of vascular malformation. In this case because the location of the fistula was identified prior to opening the dura only minimally invasive surgery was required. Direct recordings of the arterialized draining vein and the nidus of the fistula demonstrated a pathological spectrum caused by the arterial supply and the disturbed venous outflow in which a high-resistance flow pattern and low diastolic flow resembling an arterial-like flow velocity were observed. The fistula was obliterated by interruption of the draining vein, and Doppler measurements provided information on flow velocity changes in the medullary veins from an arterial to a venous pattern. The absence of any residual flow in the AVF confirmed successful hemodynamic treatment.

Intraoperative microvascular Doppler recording during surgical closure of spinal AVF is a widely available and reliable monitoring modality that helps to produce excellent clinical results.
\end{abstract}

\section{KEY WORDS • dural arteriovenous fistula • spine • ultrasonography • intraoperative monitoring}

Spinal dural AVFs are rare, insidious but curable acquired malformations that represent 3 to $4 \%$ of all spinal

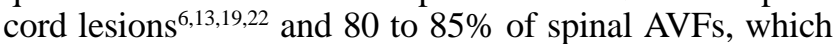
primarily involve the dorsal aspect of the thoracolumbar region. ${ }^{26}$ Typically they manifest in the fourth and fifth decades of life, more often in men than in women. These fistulas usually represent an abnormal connection between the segmental dural arterial supply of the root sleeve and the underlying medullary vein. Clinical symptoms relate to the reversal of flow in the perimedullary veins, resulting in venous hypertension. ${ }^{3}$

Because of their insidious onset, they frequently remain undiagnosed for long periods of time. Patients with these lesions can actually present with a plethora of clinical syndromes mimicking many other neurological disorders that range from multiple sclerosis to spinal cord tumors to lumbar spondylosis with neurogenic claudication. Unfor-

Abbreviations used in this paper: $\mathrm{AVF}=$ arteriovenous fistula; $\mathrm{MR}=$ magnetic resonance; $\mathrm{MV}=$ mean velocity; $\mathrm{PI}=$ pulsatility index; $\mathrm{RI}=$ resistance index . tunately, the disease can develop long before the neurological clinical signs are adequately interpreted and appropriate diagnostic and therapeutic measures are taken. The prognosis of patients with spinal dural AVFs is very serious: within 5 years of developing significant neurological deficits, the great majority of patients are paraplegic. . $^{3-5,20}$

On $\mathrm{T}_{2}$-weighted MR images an increased central cord signal intensity is observed, which can be misinterpreted to indicate myelitis, medullary ischemia, and sometimes intramedullary glioma. The emergence of gadolinium-enhanced MR imaging and MR angiography technology has helped in the differential diagnosis. , $10,21^{2}$

The most important goal in the treatment of spinal dural AVFs is to achieve complete obliteration of the fistula without aggravating spinal cord venous drainage because recurrence of the fistula is associated with an extremely high risk of progressive myelopathy.

In this article, we present a simple, noninvasive, intraoperative monitoring method, in which 16-MHz pulsed microvascular Doppler ultrasonography with a 1-mmdiameter probe, is used for easy and immediate assessment of blood flow. The goals of this report were to veri- 


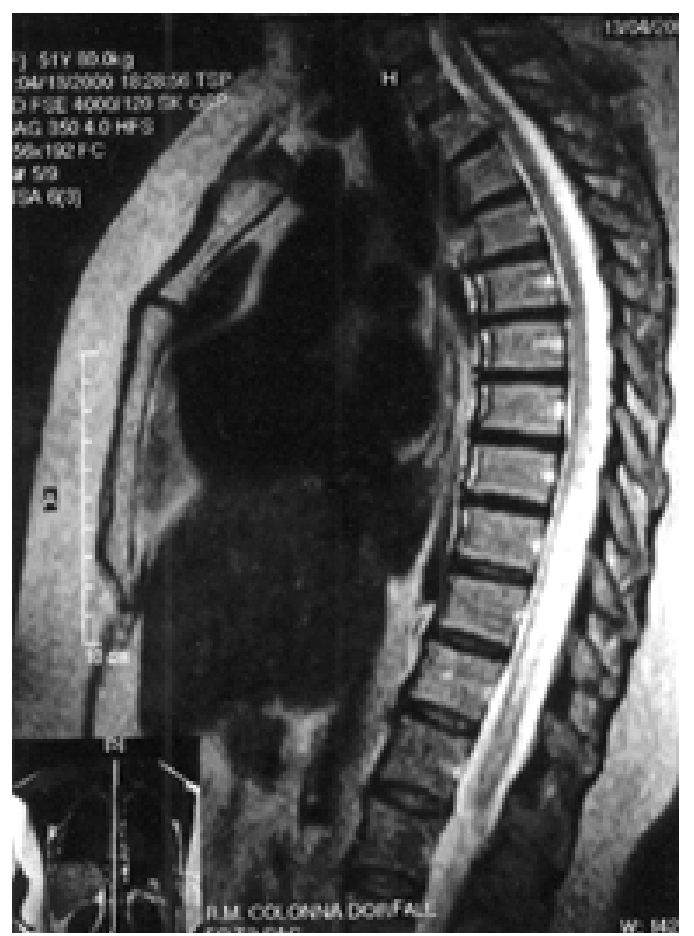

Fig. 1. Sagittal $\mathrm{T}_{2}$-weighted MR image revealing high intramedullary signal intensity at T-7 in a slightly enlarged spinal cord, associated with an intradural serpiginous vascular image suggesting the presence of an arteriovenous malformation.

fy whether it is possible to differentiate the epidural arterialized veins draining from the normal venous drainage pathway; to localize precisely the intradural fistula, thereby allowing a minimally invasive surgical approach; and to confirm the disappearance of the arterial spectrum after interruption of the fistula.

\section{CASE REPORT}

Presentation. This 51-year-old woman was admitted to our neurosurgical department with a 6-month history of progressive lower-limb weakness, bilateral lower-extremity hypesthesia and paresthesia, loss of vibratory sense, and spastic-ataxic gait.

Examinaton. A spinal MR imaging examination revealed high intramedullary signal intensity in the $T_{2}$ weighted images at the T-7 level and a slightly enlarged spinal cord simulating an intramedullary tumor (Fig. 1). Selective spinal arteriography demonstrated a dural AVF at the T7-8 level, which was fed by a single radicular artery, as well as a tortuous and ectatic venous plexus, grossly developing downward to the lumbar region (Fig. 2).

The patient underwent surgery in which the AVF was obliterated by a microvascular Doppler-assisted surgical occlusion of the arterialized vein.

The surgical treatment consisted of a limited unilateral approach: under fluoroscopic control a right-sided T7-8 hemilaminectomy and partial facetectomy were performed after making a midline skin incision. Using a microvascular Doppler device, transdural localization of the

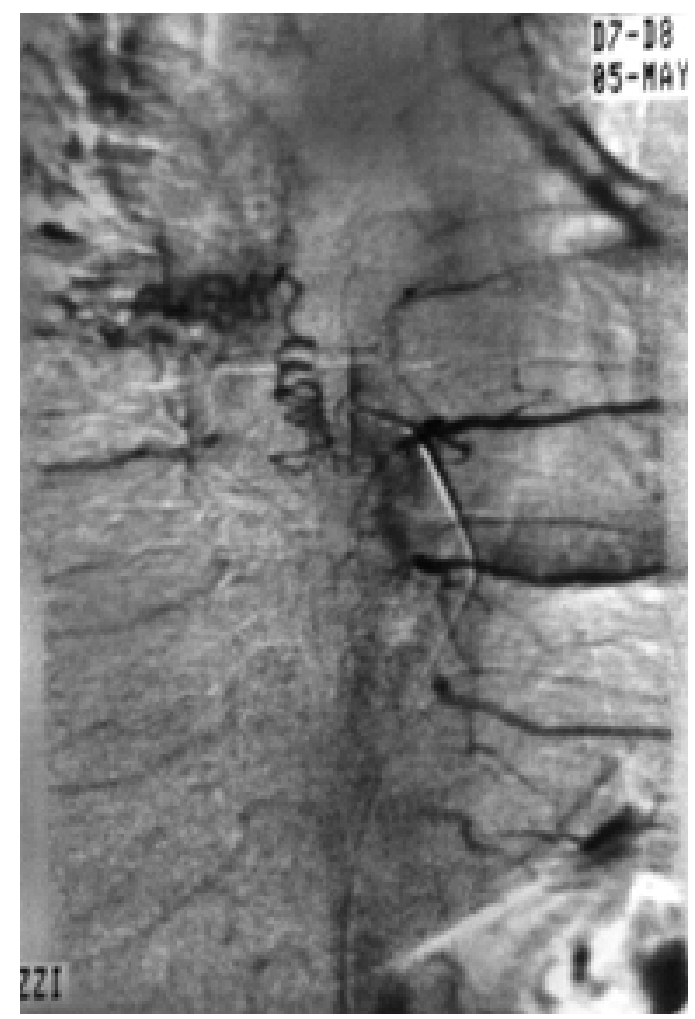

Fig. 2. Selective spinal arteriogram demonstrating a dural AVF at T7-8 that is fed by a single radicular artery and a tortuous and ectatic venous plexus, grossly developing downward toward the lumbar region.

fistula was performed (Fig. 3) allowing a minimal (3-cm) dural incision centered over the lesion. An abnormal intradural vessel was noted adjacent the right-sided T-7 nerve root. The nidus of the fistula was comprised of large, dilated, tortuous vessels developing on the dorsal surface of the cord. Additional Doppler ultrasonography measurements were acquired to identify the arterialized intradural draining vein (Figs. 4 and 5), which was then closed using vascular clips. After these procedures, the distended perimedullary veins became darker in color and collapsed within 5 to 10 minutes, and the absence of flow signal confirmed interruption of the abnormal shunt.

Click here to view video clip: A 16-MHz probe that is $1 \mathrm{~mm}$ in diameter was positioned over the dura to localize the intradural fistula. After opening the dura the probe was used to identify the arterialized draining vein, which was obliterated using vascular clip. The probe was then positioned over the clipped draining vein to verify the complete obliteration of the fistula. Additional recordings were obtained over the nidus of the fistula. The hemodynamic change from an arterial to a venous spectrum confirmed the absence of any residual flow.

Follow-Up Course. The follow-up examination showed improving motor and sensory functions at 1,3 , and 6 months postoperatively.

\section{Doppler Monitoring}

A high-frequency 16-MHz pulsed doppler device (Ex- 


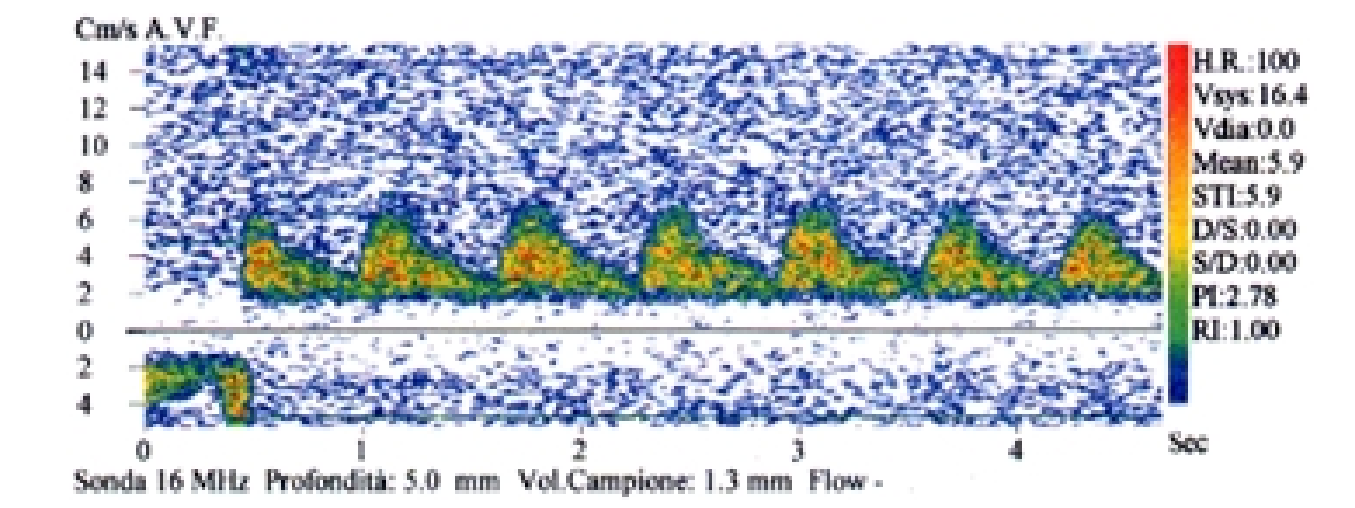

Fig. 3. Doppler measurements performed over the dura at T-7 for the localization of the intradural spinal AVF at that level. The signal, ascribable to the arterialized vein, was detected at a 5-mm depth. Blood flow velocities were $8 \mathrm{~cm} /$ second (systolic), $3 \mathrm{~cm} / \mathrm{second}$ (diastolic), and $4.7 \mathrm{~cm} / \mathrm{second}$ (MV). The RI was 0.62 and the PI 1.06. D = diastolic; $\mathrm{HR}=$ heart rate; $\mathrm{S}=$ systolic; $\mathrm{STI}=$ systolic time interval; Vdia = velocity during diastole; Vsys = velocity during systole.

plorer CVS; Diagnostic Medical Systems, Perolds, France) was used, as described by Gilsbach. ${ }^{12}$ All measurements were obtained using a sterile miniaturized probe that measured $10 \mathrm{~mm}$ in length and $1 \mathrm{~mm}$ in diameter. We attempted to keep the angle of insonation between the instrument-held probe and the vessel as low as possible so that the Doppler velocities would reflect, as closely as possible, the true blood flow velocity within the vessel. The probe position and gate depth were adjusted visually, under acoustic control of the signals, until the highest frequencies could be obtained. The measured parameters were systolic and diastolic flow velocity, the MV intended as (systolic +2 diastolic)/3, Pourcelot $\mathrm{RI}^{30}$ intended as (systolic - diastolic)/systolic, Gosling PI intended as (systolic - diastolic)/MV.

Before opening the dura, Doppler ultrasonography monitoring was first performed at the T-7 level to localize the dural AVF. At a 5-mm insonation depth, a signal indicating arterialized draining vein flow was detected on the upper side of the hemilaminectomy. The flow velocity values recorded over the dura were $8 \mathrm{~cm} / \mathrm{second}$ (systolic), $3 \mathrm{~cm} / \mathrm{second}$ (diastolic), and $4.7 \mathrm{~cm} / \mathrm{second}$ (MV). The RI was 0.62 and the PI was 1.06 (Fig. 3). After the dura was opened, an enlarged vessel was identified in the same region, as anticipated based on the extradural Doppler recording. The probe was directly applied to the enlarged vessel, and an abnormal arterial spectrum was displayed. Two more recordings were conducted. The first was recorded directly on the draining vein at an insonation depth of $2 \mathrm{~mm}$ and with a sample volume of 1.1 $\mathrm{mm}$. Recorded values were $14 \mathrm{~cm} / \mathrm{second}$ (systolic), $5 \mathrm{~cm} /$ second (diastolic), and $8 \mathrm{~cm} / \mathrm{second}(\mathrm{MV})$; the RI was 0.64 and the PI was 1.12 (Fig. 4). The second recording was conducted at the same depth on a specific vessel within the tortuous plexus overlying the spinal cord. Other recordings were obtained in the normal epidural venous plexus, which could be distinguished from the AVF because there was absence of any arterial spectrum on Doppler monitoring. After achieving complete surgical interruption of the fistula, microvascular Doppler monitoring

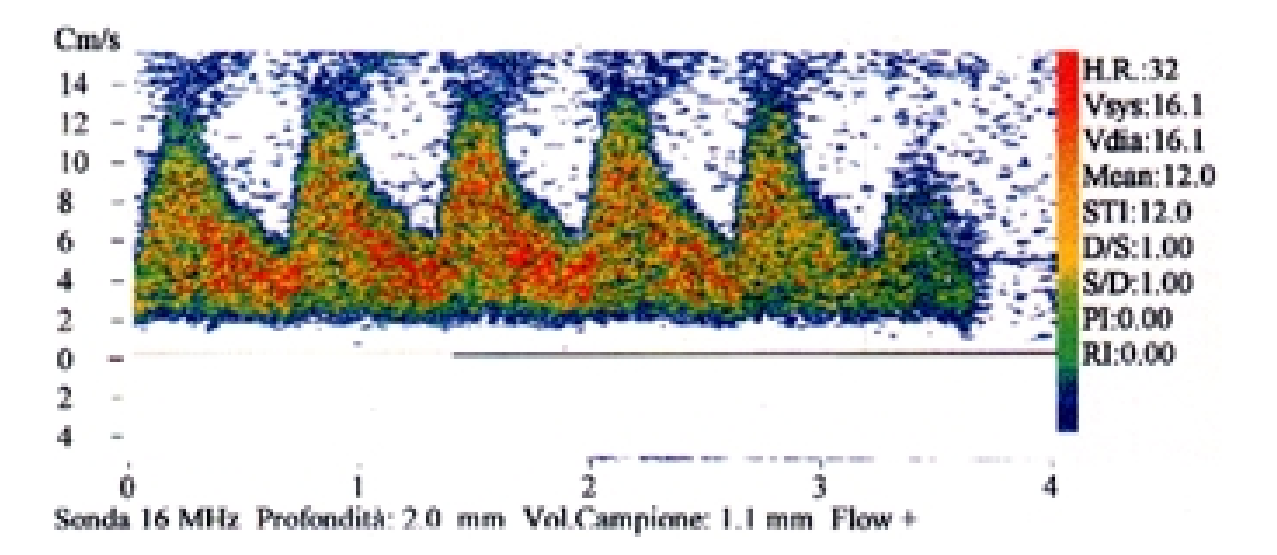

Fig. 4. Doppler measurements of the arterialized draining vein of the AVF. Recordings were performed directly over the fistula, after dural opening, at a $2-\mathrm{mm}$ insonation depth with a sample volume of $1.1 \mathrm{~mm}$. Registered flow velocities were $14 \mathrm{~cm} / \mathrm{second}$ (systolic), $5 \mathrm{~cm} / \mathrm{second}$ (diastolic), $8 \mathrm{~cm} / \mathrm{second}$ (MV); the RI was 0.64 and the PI was 1.12 . The reduced angle of insonation resulted in a higher velocity, which more closely reflects the real blood flow velocity in the examined vessel. 


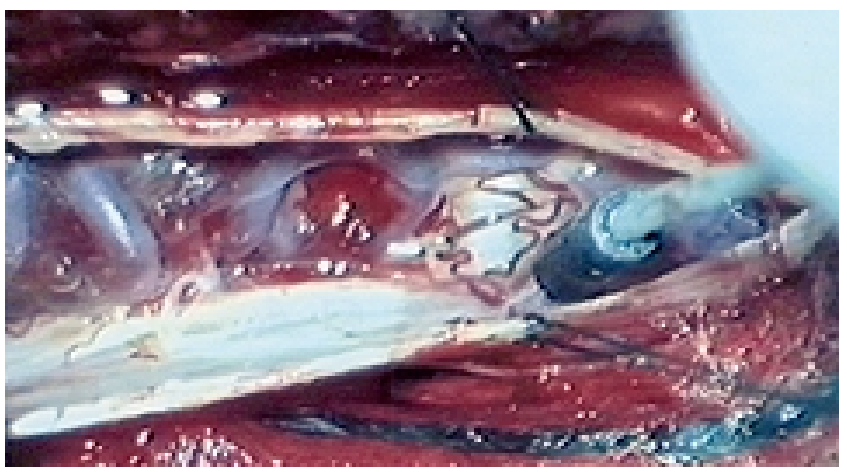

Fig. 5. Intraoperative image showing microvascular Doppler monitoring performed over the arterialized draining vein before closure of the fistula.

was performed to confirm the absence of flow velocity within the malformation and the complete disappearance of the arterial spectrum on the dorsal medullary veins.

\section{DISCUSSION}

Based on specific patterns of arterial and venous anatomy and flow velocity, dural AVFs are generally considered as type I in a four-type classification of spinal AVMs, and they represent 80 to $85 \%$ of all these vascular malformations. ${ }^{8,26}$

The pathophysiology of the spinal dural AVF was elucidated by the studies of Kendall and Logue ${ }^{19}$ in the late 1970 s, as they recognized the importance of venous hypertension. They explained that the fistula was epidural in location and usually related to a lower thoracic nerve root dural sheath. The lesion generally remains asymptomatic until drainage becomes possible, usually through a single arterialized vein, into the coronal venous plexus of the spinal cord. Because the normal veins of the coronal plexus become arterialized, the venous pressure in the spinal cord increases and pathological changes, with consequent progressive myelopathy, develop as a result of venous hypertensive ischemia., ${ }^{3,19}$ The goal in treating a spinal dural AVF is to eliminate the venous congestion of the spinal cord. Surgery or endovascular embolization is performed to interrupt of the connecting veins from the fistula to the perimedullary venous plexus. ${ }^{2,3,7,16,32,33}$ Preserving normal perfusion of the spinal cord, as well as the need to obliterate completely the AVF, cannot be emphasized enough.

The results of open surgery for spinal dural AVFs are generally excellent: complete obliteration and permanent cure have been achieved in most reported cases. A low incidence of complications and a zero mortality rate have been reported. ${ }^{13,26}$ Embolization is associated with a 60 to $90 \%$ obliteration rate. ${ }^{13,23}$ In general, surgical ligation of the intrathecal arterialized draining vein can lead to a cure with minimal complications.

The necessity of using an intraoperative monitoring system during these procedures has been previously emphasized. ${ }^{14}$ The monitoring techniques used in the past included intraoperative angiography and local invasive intravascular pressure measurement. The main advantage of the Doppler technique is that the system is noninvasive and widely available.

The Doppler effect is the change in the frequency of a soundwave when the transmitter, the receiver, and the reflecting object move in relation to one another. This change in frequency is proportional to the velocity of the movement. The Doppler effect is the difference in frequency of transmitted and received soundwaves and is proportional to blood flow velocity. ${ }^{12}$ Doppler ultrasonography recording of blood flow velocities in the extracranial arteries supplying the brain has been used to investigate cerebral hemodynamics since the first description in the early 1960s. ${ }^{17}$ The technique was then modified for the purpose of transcranial Doppler ultrasonography. ${ }^{1}$ The use of intraoperative microvascular Doppler ultrasonography for hemodynamic evaluation in cerebrovascular arteries was first described by Nornes and colleagues. ${ }^{24,25}$ By using a higher transmission frequency of $20 \mathrm{MHz}$, Gilsbach ${ }^{12}$ was able to decrease the size of the transmitter crystal and thus make the Doppler probe $3 \mathrm{~mm}$ in diameter. The actual microprobes do not exceed $1 \mathrm{~mm}$ in diameter, and they use a high transmission frequency ranging from 16 to 20 $\mathrm{MHz}$. This reduction in size allows the direct measurement of Doppler signals from otherwise inaccessible small vessels, and it permits detection of the hemodynamic effect of such operative maneuvers as the surgical closure of a vessel.

Intraoperative microvascular Doppler for spinal dural AVFs has been previously described ${ }^{11,14,18,20,29}$ and is a valid intraoperative noninvasive monitoring tool. In our experience intraoperative microvascular Doppler monitoring provides additional evidence that complete obliteration of the fistula has been achieved. In most cases, visual inspection of the draining vein is sufficient to comfirm obliteration. However, residual shunt flow may be missed by visual inspection alone.

We initially performed Doppler monitoring of the dural sheath to localize the position of the vascular malformation. It was clearly identified by its abnormal arterialized venous flow, elevated blood flow velocities, and the clear differences between the systolic and the diastolic flow values. This difference results in an increase of the resistance indices, which usually are quite low in normal venous flow. This abnormal velocity spectrum is caused by the elevated pressure in the draining vein which is produced by the arterialization of the spinal cord veins that are recruited by the malformation and/or by outflow obstruction. 3,19,31 Measurements of intravascular pressure in the draining veins have, in fact, confirmed this assumption. ${ }^{15}$ In our case it was possible to minimize the extent and invasiveness of the surgical approach because of the information provided by ultrasonography. The normal vertebral venous plexus, even if it appears enlarged, could easily be differentiated from the veins draining from the fistula, because it did not show any arterial spectrum. The dura was opened and the pathologically enlarged vein was found where expected based on extradural findings. The microvascular Doppler probe was directly applied to this vessel, and a clear pathological arterialized flow was recorded. Microvascular Doppler monitoring was also performed on redundant dorsal medullary veins.

Blood flow velocity in the AVF was characteristic of the pathological structure of this type of lesion. Due to the arterial supply and to the disturbed venous outflow, the malformation showed a spectrum characterized by high-resistance flow and low diastolic velocity. 
It is well known that the velocity patterns of arterial compared with venous flow, as detected by Doppler ultrasonography, are very different. According to Pourcelot, ${ }^{28}$ the relationship between the systolic peak flow velocity and the end-diastolic velocity of an artery is indicative of the vascular resistance in the peripheral distribution of the vessel. The RI depends mainly on the relative magnitude of the end-diastolic velocity. A low value corresponds to a low resistance, and relatively elevated end-diastolic flow velocities indicate low peripheral vascular resistance. Normally, the arterial flow is a high-resistance, pulsatile flow that is detected by Doppler examination with a characteristic velocity spectrum pattern, whereas venous flow is a low-resistance nonpulsatile flow.

The direction and velocity of the shunt flow were also examined during surgical clipping, and a dramatic hemodynamic change was detected. As the fistula was closed at is entrance within the dura, the arterialized flow completely disappeared on the medullary veins, whereas, on visual inspeciton, the vascular tangle was observed to grow darker within a few minutes.

In conclusion, intraoperative microvascular Doppler monitoring during surgical closure of spinal AVF is a widely available and noninvasive modality that may help to produce excellent clinical results.

\section{References}

1. Aaslid R, Markwalder TM, Nornes H: Noninvasive transcranial Doppler ultrasound recording of flow velocity in basal cerebral arteries. J Neurosurg 57:769-774, 1982

2. Afshar JK, Doppman JL, Oldfield EH: Surgical interruption of intradural draining vein as curative treatment of spinal dural arteriovenous fistulas. J Neurosurg 82:196-200, 1995

3. Aminoff MJ, Barnard RO, Logue V: The pathophysiology of spinal vascular malformations. J Neurol Sci 23:255-263, 1974

4. Aminoff MJ, Logue V: Clinical features of spinal vascular malformation. Brain 97:197-210, 1974

5. Aminoff MJ, Logue V: The prognosis of patients with spinal vascular malformations. Brain 97:211-218, 1974

6. Anson JA, Spetzler RF: Classification of spinal arteriovenous malformations and implications for treatment. BNI Q 8:2-10, 1992

7. Anson JA: Spinal dural arteriovenous malformation. Techn Neurosurg 2:77-85, 1996

8. Borden JA, Wu JK, Shucart WA: A proposed classification for spinal and cranial dural arteriovenous fistulous malformations and implication for treatment. J Neurosurg 82:166-179, 1995

9. Bowen BC, Fraser K, Kochan JP, et al: Spinal dural arteriovenous fistulas: evaluation with MR angiography. AJNR 16: 2029-2043, 1995

10. Chen JC, Tsuruda JS, Halbach VV: Suspected dural arteriovenous fistula: results with screening MR angiography in seven patients. Radiology 183:265-271, 1992

11. Giller CA, Meyer YJ, Batjer HH: Hemodynamic assessment of the spinal cord arteriovenous malformation with intraoperative microvascular Doppler ultrasound: case report. Neurosurgery 25:270-275, 1989

12. Gilsbach JM: Intraoperative Doppler Sonography in Neurosurgery. New York, Springer-Verlag, 1983

13. Grote EH, Bien S: Arteriovenous malformations of the spinal cord, in Youmans JR (ed): Neurological Surgery, ed 4. Philadelphia: WB Saunders, 1996, pp 1511-1530
14. Hassler W, Thron A: Flow velocity and pressure measurements in spinal dural arteriovenous fistulas. Neurosurg Rev 17: 29-36, 1994

15. Hassler W, Thron A, Groote EH: Hemodynamics of spinal dural arteriovenous fistulas. An intraoperative study. J Neurosurg 70:360-370, 1989

16. Hida K, Iwasaki Y, Goto K, et al: Results of the surgical treatment of perimedullary arteriovenous fistulas with special reference to embolization. J Neurosurg (Spine 2) 90:198-205, 1999

17. Kaneko Z: First steps in the development of the Doppler flowmeter. Ultrasound Med Biol 12:187-195, 1986

18. Kataoka H, Miyamoto S, Nagata I, et al: Intraoperative microdoppler monitoring for spinal dural arteriovenous fistulae. Surg Neurol 52:466-472, 1999

19. Kendall BE, Logue V: Spinal epidural angiomatous malformations draining into intrathecal veins. Neuroradiology 13: 181-189, 1977

20. Kerber C: Intracranial cyanoacrylate: a new catheter therapy for arteriovenous malformation. Invest Radiol 10:536-538, 1975 (Letter)

21. Mascalchi M, Bianchi MC, Quilici N, et al: MR angiography of spinal vascular malformations. AJNR 16:289-297, 1995

22. Mourier KL, Gelbert F, Rey A, et al: Spinal dural arteriovenous malformations with perimedullary drainage. Indications and results of surgery in 30 cases. Acta Neurochir 100:136-141, 1989

23. Nichols DA, Rufenacht DA, Jack CR, et al: Embolization of spinal dural arteriovenous fistula with polyvinyl alcohol particles: experience in 14 patients. AJNR 13:933-940, 1992

24. Nornes H, Grip A, Wikeby P: Intraoperative evaluation of cerebral hemodynamics using directional Doppler technique. Part 1: arteriovenous malformations. J Neurosurg 50:145-151, 1979

25. Nornes H, Grip A, Wikeby P: Intraoperative evaluation of cerebral hemodynamics using directional Doppler technique. Part 2: saccular aneurisms. J Neurosurg 50:570-577, 1979

26. Oldfield EH: Spinal vascular malformation, in Wilkins RH, Rengachary SS (eds): Neurosurgery. New York: McGrawHill, 1996, pp 2541-2558

27. Oldfield EH, Di Chiro G, Qindlen EA, et al: Successful treatment of a group of spinal cord arteriovenous malformations by interruption of dural fistula. J Neurosurg 59:1019-1030, 1983

28. Pourcelot L: Applications cliniques de l'examen Doppler transcutane. Les Colloques de l'Institute National de la Santé et de la Recherche Medicale (INSERM) 34:213-240, 1974

29. Randel S, Gooding GA, Dillon WP: Sonography of intraoperative spinal arteriovenous malformations. J Ultrasound Med 6:539-544, 1987

30. Rubin JM, Knake JE: Intraoperative sonography of a spinal cord arteriovenous malformation. AJNR 8:730-731, 1987

31. Symon L, Kuyama H, Kendall B: Dural arteriovenous malformations of the spine. Clinical features and surgical results in 55 cases. J Neurosurg 60:238-247, 1984

32. Willinsky R, terBrugge K, Montanera W, et al: Spinal epidural arteriovenous fistulas: arterial and venous approaches to embolization. AJNR 14:812-817, 1993

33. Willinsky RA, terBrugge K, Montanera W, et al: Posttreatment MR findings in spinal dural arteriovenous malformations. AJNR 16:2063-2071, 1995

Manuscript received December 18, 2000

Accepted in final form January 26, 20001.

Address reprint requests to: Domenico Gerardo Iacopino, M.D. Neurosurgical Clinic, Policlinico Universitario, Via Consolare, Valeria, 1, 98100 Messina, Italy. email: iacopino@unime.it. 